\title{
Critical behavior of an Ising system on the Sierpinski carpet: A short-time dynamics study
}

\author{
M. A. Bab, ${ }^{1,2}$ G. Fabricius, ${ }^{1,2}$ and E. V. Albano ${ }^{1}$ \\ ${ }^{1}$ Instituto de Investigaciones Fisicoquímicas Teóricas y Aplicadas (INIFTA), Facultad de Ciencias Exactas, Universidad Nacional \\ de La Plata, Sucursal 4, Casilla de Correo 16, (1900) La Plata, Argentina \\ ${ }^{2}$ Departamento de Física, Facultad de Ciencias Exactas, Universidad Nacional de La Plata, Casilla de Correo 67, (1900) La Plata, \\ Argentina
}

(Received 6 July 2004; revised manuscript received 29 October 2004; published 25 March 2005)

\begin{abstract}
The short-time dynamic evolution of an Ising model embedded in an infinitely ramified fractal structure with noninteger Hausdorff dimension was studied using Monte Carlo simulations. Completely ordered and disordered spin configurations were used as initial states for the dynamic simulations. In both cases, the evolution of the physical observables follows a power-law behavior. Based on this fact, the complete set of critical exponents characteristic of a second-order phase transition was evaluated. Also, the dynamic exponent $\theta$ of the critical initial increase in magnetization, as well as the critical temperature, were computed. The exponent $\theta$ exhibits a weak dependence on the initial (small) magnetization. On the other hand, the dynamic exponent $z$ shows a systematic decrease when the segmentation step is increased, i.e., when the system size becomes larger. Our results suggest that the effective noninteger dimension for the second-order phase transition is noticeably smaller than the Hausdorff dimension. Even when the behavior of the magnetization (in the case of the ordered initial state) and the autocorrelation (in the case of the disordered initial state) with time are very well fitted by power laws, the precision of our simulations allows us to detect the presence of a soft oscillation of the same type in both magnitudes that we attribute to the topological details of the generating cell at any scale.
\end{abstract}

DOI: 10.1103/PhysRevE.71.036139

PACS number(s): 05.50.+q, 64.60.Ht, 75.10.Hk, 02.50.-r

\section{INTRODUCTION}

In recent years, fractal structures with noninteger Hausdorff dimension $\left(d_{H}\right)$ have attracted the interest of researchers because these systems, besides serving to model natural materials such as porous rocks, aerogel, etc. [1,2], also offer the possibility of theoretically exploring systems exhibiting critical behavior close to their lower critical dimension, i.e., the larger integer dimension in which the system does not exhibit any phase transition at a finite temperature.

The first studies of phase transitions using fractal structures are those of Gefen and co-workers [3-5]. Based on renormalization methods, it has been shown that a secondorder phase transition at nonzero temperature occurs only if the fractal substrate has an infinite ramification order. Moreover, since translational symmetry is a necessary condition to proceed with dimensional perturbation [3], the disagreement between the critical exponents determined by current methods [6-11] and those obtained by continuation of $\varepsilon$ expansions to noninteger dimension [12] can be related to the topological features of the fractal structure.

It is well known that for systems with translational symmetry, the influence of the underlying structure becomes negligible at the critical point, i.e., when the correlation length is much larger than the cell spacing, and only the dimensionality, the number of components of the order parameter together with its symmetry, and the nature of the couplings concur to determine the values of the critical exponents and the corresponding universality class. However, in fractal systems, where the translational symmetry is replaced by scale invariance, the topological details of the generating cell are present at any scale and such universal behavior is said to be weak. The critical exponents and the critical temperature depend not only on $d_{H}$, but also on the connectivity and lacunarity of the fractal [13]. A direct quantitative study of topological effects has been recently published [14].

Most of the previously cited studies are based on the same type of fractal, i.e., the Sierpiński carpet (SC), which has an infinite ramification order. Although the same kind of magnetic interaction (Ising model) has been considered, these previous studies yield controversial results. Table I summarizes the list of published results for the case of the twodimensional SC with $d_{H}=1.8927$, where the generating cell is built by segmenting a square into nine subsquares and removing the central one, so that this fractal is termed $\mathrm{SC}(3,1)$. In particular, different values have been obtained by different authors for the critical temperature $\left(T_{c}\right.$, see Table I), which in most cases is lower than $T_{c}$ of the Ising model in two dimensions (2D). Also, a considerable scattering in the data corresponding to the critical exponents $\beta / \nu$ and $\gamma / \nu$ can be observed. It should also be noticed that Monte Carlo simulations and finite-size scaling (FSS) may predict either $d_{H}>d_{\text {eff }}[7,9]$ or $d_{H}<d_{\text {eff }}[8]$.

One of the reasons explaining the discrepancies reported in the calculation of $T_{c}$ is most likely related to the location of the spins on the fractal (see Table I), which could be at the vertices $[3,5,6,11]$ or at the centers $[7-10]$ of the squares. Consequently the mean number of nearest neighbors per site is not the same. In addition, for the former the number of spins as a function of the cell size does not follow a power law and consequently $d_{H}$ should not be expected to enter in the description of the critical phenomena of such systems. Another reason causing discrepancies is related to finite-size effects, since scaling analyses reveal very strong scaling corrections for dimensions smaller than $d=2$ [7-9]. Monceau 
TABLE I. List of critical temperatures and critical exponents reported in the literature for the Ising model in the $\mathrm{SC}(3,1)$ fractal. The methods used to obtain the data are real space group renormalization (RSGR); Monte Carlo simulations and finite-size scaling (MC-FSS); Monte Carlo renormalization group (MC-RNG); Monte Carlo simulations and the slope method (MC-slope) [10]. The index $k$ indicates the generation of fractal used. The boundary conditions $(\mathrm{BC})$ used are either periodic $(\mathrm{P})$ or free $(\mathrm{F})$.

\begin{tabular}{cccccccccc}
\hline \hline Reference & $T_{c}$ & $\nu$ & $\beta / \nu$ & $\gamma / \nu$ & $d_{\text {eff }}$ & $k$ & BC & Method & Spin location \\
\hline$[6]$ & 2.06 & 1.12 & & & & & P & RSRG & Vertices \\
{$[3]$} & 3.12 & & & & & & F & RSRG & Vertices \\
{$[8]$} & $1.482(15)$ & $1.565(10)$ & $0.0815(30)$ & $1.76(1)$ & $1.923(16)$ & 7 & $\mathrm{P}$ & MC-FSS & Center \\
{$[8]$} & 1.482 & $1.73(3)$ & $0.147(9)$ & $1.625(20)$ & $1.919(28)$ & 7 & $\mathrm{~F}$ & MC-FSS & center \\
{$[7]$} & 1.481 & $1.70(1)$ & $0.080(1)$ & $1.730(1)$ & $1.890(2)$ & 7 & $\mathrm{P}$ & MC-FSS & Center \\
{$[9]$} & $1.4795(5)$ & & $0.075(10)$ & $1.732(4)$ & $1.882(24)$ & 8 & $\mathrm{P}$ & MC-FSS & Center \\
{$[10]$} & $1.4992(11)$ & & & & $\simeq 1.7$ & 6 & $\mathrm{P}$ & MC-Slope & Center \\
{$[7]$} & $1.479546(16)$ & & & & & & $\mathrm{P}$ & MC-RNG & Center \\
Ising & 2.269 & 1 & 0.125 & 1.75 & 2 & & Exact & \\
\hline \hline
\end{tabular}

and Perreau [9] have noticed that discrepancies with standard finite-size scaling methods could also be due not only to the operation of pure (genuine) finite-size effects, but also to a topological contribution to scaling corrections [9]. They have also pointed out that the hyperscaling law $d_{\text {eff }}=2 \beta / \nu+\gamma / \nu$ should remain valid for $d_{\mathrm{eff}}=d_{H}$ and the susceptibility should follow the expected power-law behavior, which allows calculation of the ratio of exponents $\gamma / \nu$ and the anomalous dimension exponent $\eta$ in a reliable way [15]. In addition, these authors have shown the agreement of their results with those determined by Monte Carlo renormalization group techniques [16].

On the order hand, Pruessner et al. [10] have questioned the validity of FSS studies on fractal structures. In fact, they have pointed out that each segmentation step represents a new thermodynamic system and cannot be treated as a scaled version of the previous one. In order to avoid this shortcoming, the authors have proposed the slope method [10]. The critical exponents obtained using this method suggest that $d_{\text {eff }}$ is smaller than $d_{H}$ [10], in agreement with the $4-\varepsilon$ renormalization group prediction. Furthermore, the obtained critical temperature is higher than those reported by other authors by using FSS (see Table I).

Another useful approach to obtain independent estimations for the critical temperature and the critical exponents is given by the short-time dynamics (STD) [17] method (for available results see rows $1-3$ of Table II). By performing simulations using both the fourth and fifth segmentation steps, Pruessner et al. [10] have reported substantial differences between the critical temperature estimated by the STD method (see Table II) and the data corresponding to the FSS approach (see Table I). Furthermore, the exponents $\nu z, \gamma$, and $\beta$ (see also Table II) strongly depend on the segmentation step. In addition, Zheng and $\mathrm{Li}[11]$ have determined the exponent $\theta$ of the initial increase in magnetization which seems to be slightly greater than the figure accepted for the 2D Ising model. However, this determination has to be taken with caution because spins are located at the vertices of the fractal and this approach is expected to give an inaccurate estimation of $T_{c}$, as has already been discussed.

In view of the scattering of the available data for the critical temperature and critical exponents, the aim of this paper is to study the critical behavior of the Ising model on the Sierpiński carpet fractal structure $\mathrm{SC}(3,1)$ using the short-time dynamics approach. In order to achieve this goal, we have used a segmentation step bigger than in a previous STD study of this system [10]. Furthermore, we have confirmed that the time dependence of the magnetization follows a power-law behavior for times two orders of magnitude larger than in a previous STD study [10]. Additionally, we have determined $T_{c}$ and the complete set of critical exponents starting the STD studies with two different initial con-

TABLE II. List of critical temperatures and critical exponents reported in the literature for the Ising model in the SC(3,1) fractal and obtained using Monte Carlo simulations and a short-time dynamics analysis. PW indicates the present work. The boundary conditions used are periodic and the index $k$ indicates the generation of the fractal used.

\begin{tabular}{ccccccccc}
\hline \hline Reference & $T_{c}$ & $\nu z$ & $\gamma$ & $\beta$ & $\theta$ & $z$ & $k$ & Spin location \\
\hline$[11]$ & $2.033(4)$ & & & & $0.211(3)$ & $2.38(4)$ & 7 & Vertices \\
{$[10]$} & $1.5266(11)$ & $3.06(11)$ & $1.959(32)$ & $0.1154(29)$ & & & 4 & Center \\
{$[10]$} & $1.5081(12)$ & $3.21(15)$ & $2.048(49)$ & $0.120(55)$ & & & 5 & Center \\
PW & $1.4945(50)$ & $3.546(12)$ & $2.22(1)$ & $0.121(5)$ & $0.1815(6)$ & $2.55(1)$ & 6 & Center \\
Ising & 2.269 & $2.165[24]$ & 1.75 & 0.125 & $0.191[24]$ & $2.165[24]$ & & \\
\hline \hline
\end{tabular}


ditions: (i) a fully ordered initial state (ground state configuration corresponding to $T=0$ ), and (ii) a fully disordered initial state corresponding to $T=\infty$. Self-consistency of the results was also carefully checked. On the other hand, we have also obtained the value of the exponent $\theta$ related to the initial increase in magnetization at an early time, which have not been previously determined for spins placed at the center of the occupied subsquares of the fractal. Finally, the dynamic exponent $z$ was evaluated for this fractal, by means of two independent methods using the Binder cumulant and the critical scaling of time correlation functions.

The STD approach has shown to be a powerful tool in the study of critical phenomena because the critical exponents can be determined before the critical slowing down of the dynamics takes place and they are free from finite-size corrections, provided that the correlation length $\xi(t)$ is always smaller than the system size $L[\xi(t) \ll L][18,19]$. However, in the case that we are interested in, namely, $\mathrm{SC}(3,1)$, an intrinsic kind of finite-size effect due to the segmentation step $k$ of the fractal can be observed, as is discussed in detail below. Furthermore, the STD analysis is particularly useful since the application of FSS to obtain $T_{c}$ is questionable for segmentation steps smaller than 6 [10], which are the typical sample sizes that one is forced to use in practical calculations due to computing limitations [7-9].

The outline of this paper is the following. In Sec. II we describe the magnetic model and the underlying fractal structure, in Sec. III we briefly recall the main features of the short-time dynamics method, and in Sec. IV, the results obtained from the dynamic simulations are presented. Finally, our conclusions are stated and discussed in Sec. V.

\section{THE ISING MODEL ON THE SIERPIŃSKI CARPET}

The $\operatorname{SC}(b, c)$ is obtained as follows: for each segmentation step $(k)$, a square of length $L$ is segmented into $b^{2}$ subsquares and $c^{2}$ subsquares are deleted from the center of the initial square; then the segmentation process is iterated on the remaining subsquares. Figure 1 shows a sketch of the $\mathrm{SC}(3,1)$, which is used in the present work, corresponding to the $k=3$ segmentation step. In the limit $k \rightarrow \infty$ the mathematical fractal $\mathrm{SC}(b, c)$ is obtained, and the Hausdorff dimension is given by $d_{H}=\ln \left(b^{2}-c^{2}\right) / \ln b$. In the case of the $\operatorname{SC}(3,1)$ the deviation of the mean number of nearest neighbors from that corresponding to the thermodynamic limit (mathematical fractal), using periodic boundary conditions, as determined by the transfer-matrix method, becomes negligible for $k \geqslant 6$ [9].

As mentioned above, this fractal has an infinite ramification order, which implies that the Ising model should exhibit a second-order phase transition at finite temperature. Spins were placed at the center of the occupied subsquares. Consequently the number of spins increases as a power law of the lattice size, and the exponent is given by $d_{H}$.

The Hamiltonian of the system is given by

$$
H=-J \sum_{\langle i, j\rangle} s_{i} S_{j}
$$

where $s_{i}$ assumes the values \pm 1 , the sum runs over all

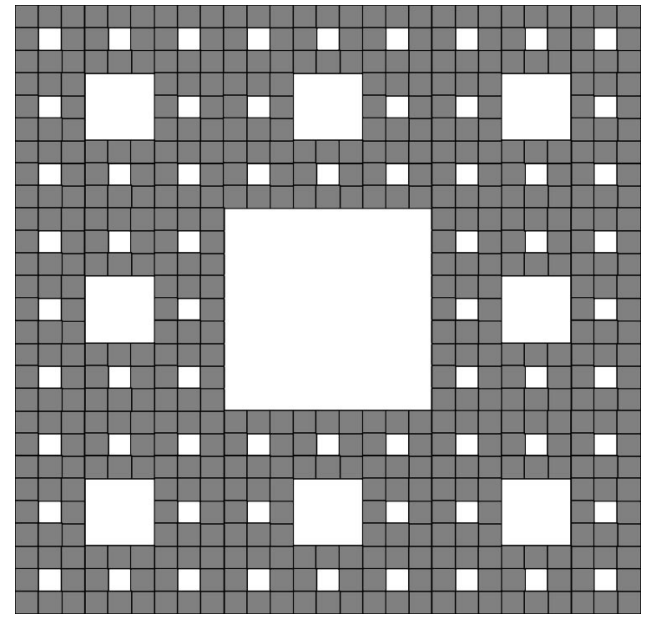

FIG. 1. Sketch of the Sierpinski carpet $\mathrm{SC}(3,1)$ iterated up to $k=3$ segmentation step. Spins are placed at the centers of the filled squares.

interacting nearest-neighbor pairs of spins, and the exchange coupling constant $J$ is positive (ferromagnetic interactions).

\section{SHORT-TIME DYNAMICS APPROACH FOR CRITICAL PHENOMENA}

According to field theoretical calculations [17], if a magnetic system at high temperature, with a small magnetization $m_{0}$, is suddenly quenched to the critical temperature, it may exhibit a universal dynamic evolution, which sets in right after a time scale $t_{\text {mic }}$. It is expected that $t_{\text {mic }}$ should be large in the microscopic sense, but still very small in the macroscopic sense necessary for equilibration. This STD approach is free of the critical slowing down since the spatial correlation length is still small within the short-time regime, even at (or near) the critical point [17].

The $k$ th moments of the magnetization are given by $[18,19]$

$$
M^{k}\left(t, \tau, L, m_{0}\right)=b^{-k \beta / \nu} M^{k}\left(b^{-z} t, b^{1 / \nu} \tau, b^{-1} L, b^{x_{0}} m_{0}\right)
$$

where $\beta$ and $\nu$ are the order parameter and the correlation length critical exponents, $z$ is the dynamic critical exponent, $\tau$ is the reduced temperature, $L$ is the system size, $x_{0}$ is the scaling dimension of the initial magnetization, and $b$ is a scaling factor. For large $L$, at the critical point $\tau=0$, and for $m_{0} \ll 1$, from the scaling form given by Eq. (2) one derives the initial increase in magnetization, obtaining $[18,19]$

$$
M(t)=\left[\left\langle\frac{1}{N} \sum_{i=1}^{N} s_{i}\right\rangle\right]=m_{0} t^{\theta} F\left(t^{\theta+\beta / \nu z} m_{0}\right)
$$

where $\langle\cdots\rangle$ denotes the averages taken over spin configurations and $[\cdots]$ corresponds to averages taken over different samples with equivalent initial conditions. Here, $\theta=\left(x_{0}\right.$ $-\beta / \nu) / z$, and the scaling function behaves as $F(x) \sim 1$ for $x \rightarrow 0$ and $F(x) \sim 1 / x$ for $x \rightarrow \infty$. It should be noticed that the time scale for this initial increase is of the order of $t_{0}$ $\sim m_{0}^{-z / x_{0}} . \theta$ and $x_{0}$ are the exponents of the initial increase 
and the scaling dimension of the order parameter. Since both exponents are related, one of them can be considered as an additional nontrivial critical exponent $[18,19]$. Performing simulations for different values of the initial magnetization and extrapolating the results to $m_{0}=0$, the exponent $\theta$ can be obtained. Other interesting observables are the second moment of the magnetization $\left[M^{2}(t)\right]$ and the autocorrelation [A(t)], which for $m_{0}=0$ and $\tau=0$ should behave according to the following power-law scaling relationships:

$$
M^{2}(t)=\left[\left\langle\left(\frac{1}{N} \sum_{i=1}^{N} s_{i}\right)^{2}\right\rangle\right] \propto t^{\left[d_{\mathrm{eff}} \mathrm{z} z-2 \beta / v z\right]}
$$

and

$$
A(t)=\left[\left\langle\frac{1}{N} \sum_{i=1}^{N} s_{i}(t) s_{i}(0)\right\rangle\right] \propto t^{-\lambda} \quad \text { with } \lambda=\frac{d_{\mathrm{eff}}}{z}-\theta,
$$

respectively.

Another important process that can be measured is the dynamic relaxation from a completely ordered state (with $m_{0}=1$ ), which corresponds to a ground state configuration at $T=0$, to $T_{c}$. In this case, the magnetization, the logarithmic derivative of the magnetization with respect to $\tau$, and the second-order Binder cumulant [24] should behave according to

$$
\begin{gathered}
M(t) \propto t^{-\beta / v z}, \\
V_{\tau}(t)=\left.\partial_{\tau}[\ln M(t, \tau)]\right|_{\tau=0} \propto t^{1 / v z},
\end{gathered}
$$

and

$$
U(t)=\frac{M^{2}(t)}{[M(t)]^{2}}-1 \propto t^{d_{\mathrm{eff}}},
$$

respectively. For $T \neq T_{c}$, but within the critical region, the power-law behavior is modified by a scaling function, which for the magnetization is given by $M\left(t^{1 / v z} \tau\right)$. This fact can be used to determine the critical temperature from the localization of the optimal power-law behavior.

Summing up, the STD scaling study of a given system performed by starting from two extreme initial states, i.e., a completely ordered one and a completely disordered one, is sufficient to determine both the critical temperature and the set of relevant critical exponents in a self-consistent fashion [18-20].

\section{NUMERICAL RESULTS}

\section{A. Details on the simulations}

Monte Carlo simulations of the Ising model on the $\mathrm{SC}(3,1)$ were performed for the segmentation step $k=6$ ( $L$ $=729$ with 262144 spin sites) using periodic boundary conditions, and starting either from an ordered state or from a disordered state with zero or a small initial magnetization. In the latter case the initial magnetization $\left(m_{0}\right)$ was obtained from a disordered configuration (of zero magnetization) by

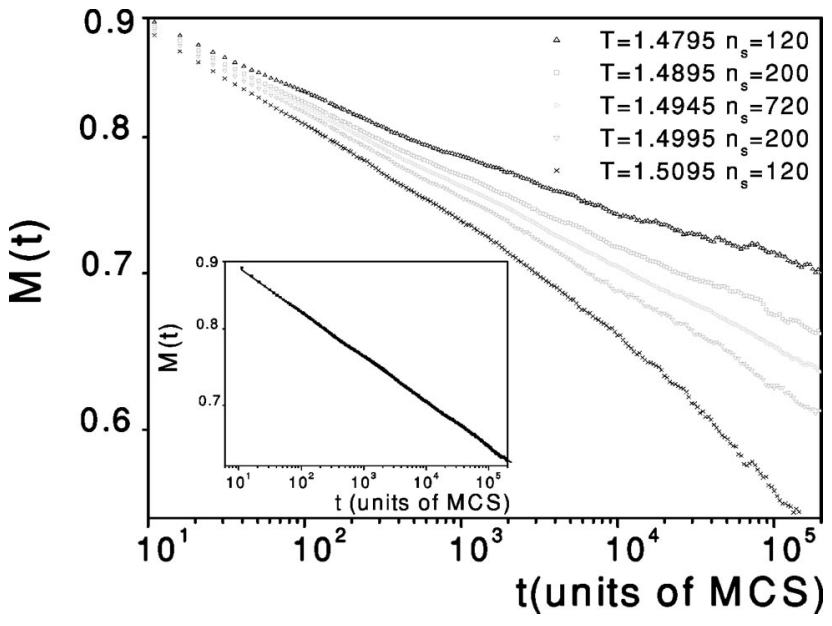

FIG. 2. Log-log plots of magnetization versus time obtained starting from ordered initial conditions $\left(m_{0}=1\right)$. The different temperatures used and the number of samples considered are also shown in the figure. The inset shows the log-log plot of $M(t)$ versus $t$ that gives the best fit of Eq. (6) and is assumed to correspond to the critical temperature $T_{c}=1.4945$.

flipping a definite number of spins at randomly chosen sites.

In order to implement the time evolution, the system is updated by using the Metropolis algorithm. The well tested [21] Marsaglia-Zanan pseudorandom number generator is used throughout the simulations. The time unit, defined as a Monte Carlo time step (MCS), involves the update of a number of spins that corresponds to all spin sites of the sample. In this way, during one MCS each spin is updated once, on average. Simulations starting from a disordered (ordered) state are carried out up to 2000 MCS (200 000 MCS).

The magnetization, the autocorrelation, and the second moment of the magnetization were averaged over a number $n_{s}$ of samples with equivalent initial configurations. In addition, the time evolution of the ordered state was also studied for the segmentation steps $k=3,4,5$ in order to apply a FSS method that allowed us to obtain an estimation of the dynamic exponent $z$. In order to estimate the error bars of the evaluated exponents we have used a variant of the blocking method [22] fitting the time dependence of each observable for independent sets of measurements having the same statistic.

\section{B. Simulation of the dynamic evolution starting from the ordered state}

According to our experience, the determination of the critical temperature and exponents is more accurate when the simulations start from the ordered state. In fact, in this case the magnetization is large and decreases slowly during time evolution and therefore statistical fluctuations are less prominent. Figure 2 shows the decay of the magnetization obtained at different temperatures for $k=6$. The critical temperature is determined by finding the smallest standard deviation from the power law given by Eq. (6), which yields $T_{c}(k=6)$ $=1.4945(50)$, where the error bar is assessed by considering the closest pair of temperatures that present noticeable but 
TABLE III. List of critical exponents determined from the time dependence of the magnetization [second column, see Eq. (6)], Binder's cumulant [third column, see Eq. (8)], the logarithmic derivative of magnetization [fourth column, see Eq. (7)], and the susceptibility (fifth column). Data obtained starting the simulations from an ordered initial state and for the $k=6$ generation of the fractal. Slightly different exponents are obtained by performing the fits after disregarding different initial time intervals $\left(t_{\min }\right)$, as listed in the first column.

\begin{tabular}{ccccccc}
\hline \hline$t_{\min }(\mathrm{MCS})$ & $\beta / \nu z$ & $d_{\mathrm{eff}} / z$ & $1 / \nu z$ & $\gamma / \nu z$ & $\beta$ & $\gamma$ \\
\hline 20 & $0.03406(6)$ & $0.697(3)$ & $0.285(2)$ & $0.630(3)$ & $0.119(9)$ & $2.21(2)$ \\
100 & $0.03412(7)$ & $0.693(2)$ & $0.282(1)$ & $0.626(2)$ & $0.121(5)$ & $2.22(1)$ \\
150 & $0.03413(7)$ & $0.694(2)$ & $0.282(2)$ & $0.627(2)$ & $0.121(9)$ & $2.22(2)$ \\
\hline \hline
\end{tabular}

small standard deviations. This figure is in good agreement with determinations performed by means of Monte Carlo simulations analyzed by using the slope method [10]. Also, acceptable agreement with the value obtained by means of the STD analysis of Monte Carlo data is found (see Table II). However, a careful inspection of the data shows a systematic decrease in $T_{c}$ when the segmentation step is increased, suggesting that our result could be taken as an upper bound.

The exponent determined by fitting Eq. (6) [see the inset of Fig. 2] is listed in Table III (second column). Our estimation, given by $\beta / \nu z=0.0341(1)$, is slightly smaller than the figures obtained by using the exponents listed in Table II, which correspond to STD studies for $k=4$ and 5, namely, $\beta / \nu z=0.0377$ and 0.0374 [10], respectively. So our finding is consistent with a systematic decrease in $\beta / \nu z$ that is observed when $k$ is increased.

The logarithmic derivative of the magnetization with respect to $\tau$ is evaluated by taking the difference between the values of $M(t)$ at two temperatures close to $T_{c}$. The result of this calculation, obtained taking $T_{1}=1.4795$ and $T_{2}=1.5095$, is shown in Fig. 3. From this figure it follows that the powerlaw behavior expected from Eq. (7) is obtained after $t=70$ MCS, and the corresponding exponent is listed in Table III. It should be noticed that using $T_{1}=1.4895$ and $T_{2}=1.4995$ one also obtains the same critical exponent (within error bars) but the data are more noisy. From the data shown in Table III it follows that $\nu z=3.546$, which is significantly larger than the exponent reported for $k=5$, namely, $\nu z=3.21$ [10].

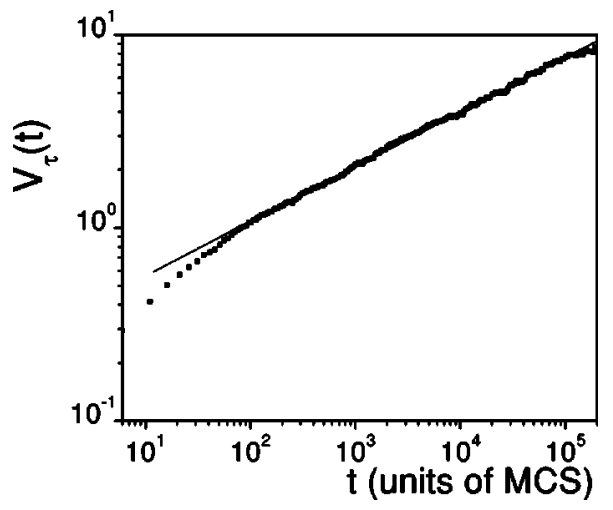

FIG. 3. Log-log plot of the logarithmic derivative of magnetization versus time obtained at criticality, starting from ordered initial conditions $\left(m_{0}=1\right)$. The full line corresponds to the best fit obtained for $t>100 \mathrm{MCS}$, according to Eq. (7).
Figure 3 shows the determination of the exponent $d_{\mathrm{eff}} / z$ (see the results in Table III) from the time dependence of the Binder cumulant, according to Eq. (8). Considering that the susceptibility is given by $\chi(t) \propto U(t)[\langle M(t)\rangle]^{2} \propto t^{\gamma / v z}$, the exponent $\gamma / \nu z$ can also be obtained (see inset in Fig. 3 and Table III). Notice that the above relationships were obtained assuming that the hyperscaling law $d_{\mathrm{eff}}=2 \beta / \nu+\gamma / \nu$ holds.

Simulations started from the ordered state also allow the self-consistent determination of the order parameter critical exponent $\beta$ and the exponent $\gamma$ of the susceptibility using the exponents listed in the second and fourth columns, and in the fifth and fourth columns of Table III, respectively. The obtained results are also listed in Table III. The trend of the data for the exponent $\gamma$, namely, a systematic increase with $k$, is consistent with the observations reported by Pruessner et al. [10], which are listed in Table II for the sake of comparison. However, $\beta$ appears to be less sensitive to the change of the segmentation step. It is worth mentioning that our best estimation of the order parameter critical exponent given by $\beta$ $=0.121(5)$ is very close to the exact value corresponding to the Ising model in $d=2(\beta=0.125)$. However, $\gamma$ is clearly greater in the case of the fractal substrate.

\section{Simulations of the dynamic evolution starting from a disordered state}

Figure 4 shows the initial increase in magnetization, observed for different values of the initial (small) magnetization $\left(m_{0}\right)$, and obtained after quenching the system to $T_{c}$ when the simulations started from the disordered state corresponding to $T=\infty$.

Within the time regime considered (20-2000 MCS), the magnetization always increases and the data can be fitted to a power law with critical exponent $\theta$, as expected from Eq. (3). Nevertheless, a soft curvature of the data can be observed for larger times due to the fact that $m_{0}$ is finite and the power law is actually expected to hold in the $m_{0} \rightarrow 0$ limit. So, in order to determine the critical exponent we performed a fit of the data within the time interval 20-100 MCS. As can be observed in the inset of Fig. 4, the exponents show a weak dependence on $m_{0}$. Then the exponent $\theta$ was evaluated by a linear extrapolation to $m_{0}=0$, yielding $\theta=0.1815(6)$. So, according to our results, the exponent $\theta$ for the $\operatorname{SC}(3,1)$ fractal appears to be slightly smaller than the accepted value for the Ising model in $d=2$, given by $\theta=0.191$ [23]. 


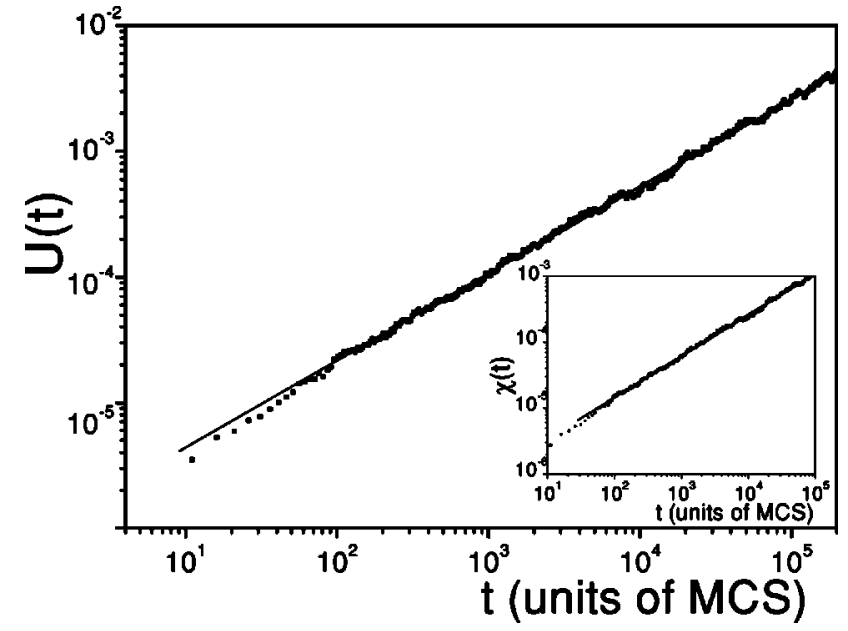

FIG. 4. Log-log plot of Binder's cumulant versus time obtained at criticality, starting from ordered initial conditions $\left(m_{0}=1\right)$. The inset shows the zero field susceptibility $(\chi)$ versus time. The full lines correspond to the best fits obtained for $t>100 \mathrm{MCS}$.

Figure 5 shows that the evolution from a disordered state, with $m_{0}=0$, of the second moment of the magnetization has a weak dependence on temperature. This shortcoming hinders an independent estimation of $T_{c}$ based on these measurements. However, by using the value of $T_{c}$ obtained by means of simulations started from the ordered state, it is possible to evaluate the critical exponent of the second moment according to Eq. (4), as shown in the inset of Fig. 5. The obtained value is listed in Table IV.

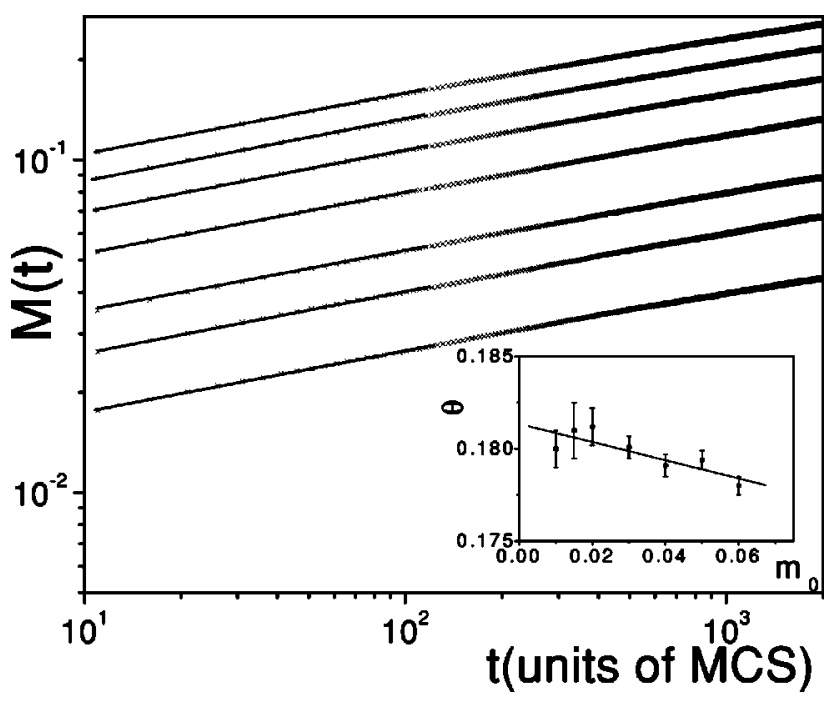

FIG. 5. Log-log plots of magnetization versus time obtained at criticality, starting from disordered initial conditions slightly modified to obtain different values of the initial magnetization $m_{0}$. Data corresponding to $k=6$ and different values of $m_{0}$, which from top to bottom are $0.06,0.05,0.04,0.03,0.02,0.015$, and 0.01 , respectively. The full lines correspond to the best fits obtained for $20 \leqslant t$ $\leqslant 100 \mathrm{MCS}$, according to Eq. (3). Data obtained by averaging over 4000-10 000 different samples, depending on $m_{0}$. The inset shows the dependence of $\theta$ on the initial magnetization $m_{0}$ that allowed us to extrapolate the exponent $\theta\left(m_{0} \rightarrow 0\right)=0.1815(6)$. More details in the text.
TABLE IV. List of critical exponents determined from the dynamic behavior of the second moment of the magnetization [second column, see Eq. (4)] and autocorrelation [third column, see Eq. (5)]. Data obtained starting the simulations from disordered initial states with $m_{0}=0$ and for $k=6$. The estimations of $d_{\mathrm{eff}} / z$ listed in columns 4 and 5 are obtained by using the autocorrelation data (third column) and the determined value of $\theta=0.1815$, and using the exponents listed in the second column in combination with the exponents listed in the second column of Table III, respectively.

\begin{tabular}{ccccc}
\hline \hline$t_{\min }(\mathrm{MCS})$ & $d_{\mathrm{eff}} / z-2 \beta / \nu z$ & $d_{\mathrm{eff}} / z-\theta$ & $d_{\mathrm{eff}} / z$ & $d_{\mathrm{eff}} / z$ \\
\hline 20 & $0.665(2)$ & $0.518(2)$ & $0.699(2)$ & $0.699(2)$ \\
100 & $0.648(2)$ & $0.514(2)$ & $0.695(2)$ & $0.682(2)$ \\
150 & $0.646(2)$ & $0.512(3)$ & $0.693(3)$ & $0.680(2)$ \\
\hline \hline
\end{tabular}

On the other hand, the decay of the autocorrelation function (see Fig. 6) slightly depends on $T$, allowing us to confirm our estimation, namely, $T_{c}=1.4945(50)$, and the corresponding error bars, already evaluated using simulations started from ordered configurations. The exponent $\lambda=d_{\mathrm{eff}} / z$ $-\theta$ evaluated by fitting the data at criticality (see inset of Fig. 6) is also listed in Table IV. It is worth mentioning that by inserting the value of $\theta$ already determined in the exponent of the autocorrelation function, one can also calculate $d_{\text {eff }} / z$, as listed in the fourth column of Table IV. The obtained results are in full agreement with the determination performed by starting simulations from ordered states (see Table III).

Also, inserting the exponent $\beta / \nu z$ determined by means of simulations started from the ordered state (see the second column of Table III) in the expression of the exponent of the second moment of the magnetization, given by $d_{\text {eff }} / z$ $-2 \beta / \nu z$, one can obtain an additional estimation of $d_{\text {eff }} / z$, as

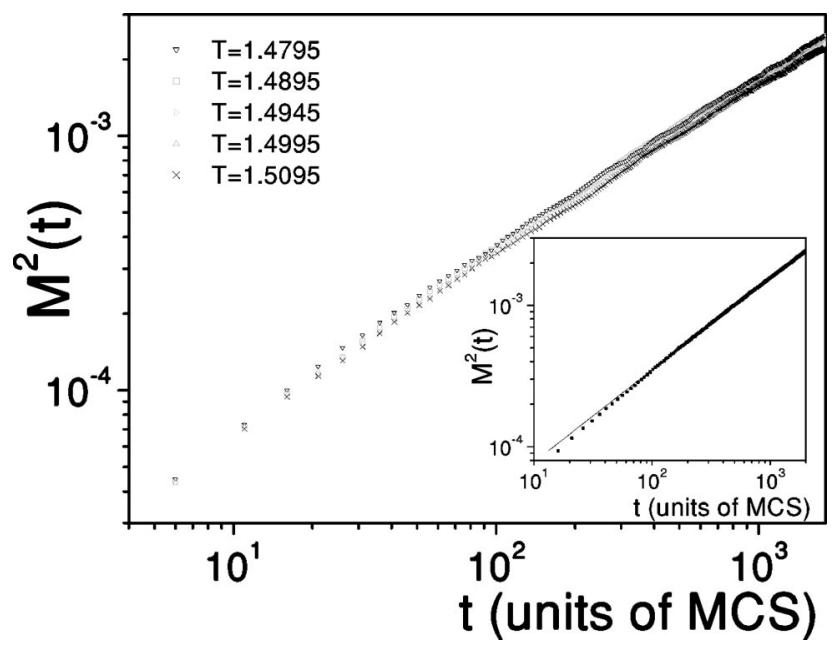

FIG. 6. Log-log plots of the second moment of magnetization versus time, obtained at different temperatures (listed in the figure), starting from disordered initial conditions with $m_{0}=0$. Data obtained by taking $k=6$. The inset shows the dependence of $M^{2}$ on time obtained at criticality $\left(T_{c}=1.4945\right)$, where the exponent $d_{\text {eff }} / z-2 \beta / \nu z$ is obtained by fitting the data according to Eq. (4). See also Table IV. Data obtained by averaging over 8000 different samples. 


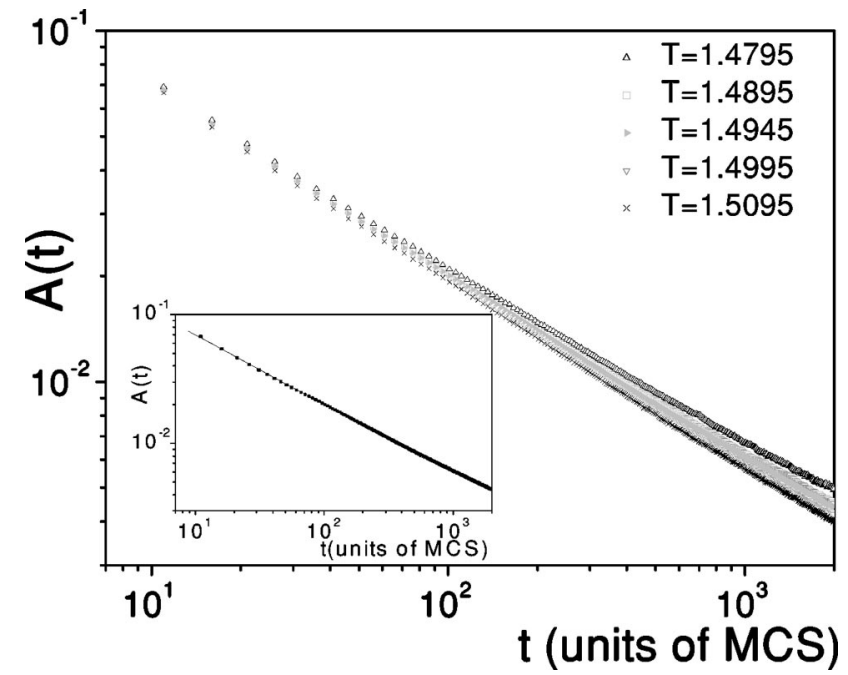

FIG. 7. Log-log plots of autocorrelation versus time, obtained at different temperatures (listed in the figure), starting from disordered initial conditions with $m_{0}=0$ and $k=6$. The inset shows the dependence of autocorrelation on time obtained at criticality $\left(T_{c}\right.$ $=1.4945)$, where the exponent $\lambda=d_{\mathrm{eff}} / z-\theta$ is obtained by fitting the data according to Eq. (5). See also Table IV. Data obtained by averaging over 8000 different samples.

listed in the fifth column of Table IV, which is slightly smaller than the estimations already performed by means of different procedures. We attribute this small difference to the propagation of errors in the evaluation of exponents by combining results from different measurements.

\section{Determination of the dynamic exponent $z$}

Although the application of FSS techniques to the evaluation of critical exponents in systems with fractal structure is questionable, as we have already mentioned in the Introduction, we used it at this point in order to obtain a first estimation of dynamic exponent $z$. However, we will perform a critical analysis of the obtained results and subsequently we will perform a second (more accurate) estimation of $z$. We carried out simulations up to $10^{4}$ MCS for segmentation steps $k=3,4,5$, and 6 ( $L=27,81,243$, and 729 , respectively) and determined the dynamic exponent $z$ using a FSS analysis of the Binder cumulant. In fact, right at $T_{c}$, the dynamic exponent $z$ can be determined from the Binder cumulant according to the following scaling relation:

$$
U\left(t, L_{1}\right)=U\left(t b^{z}, L_{2}\right),
$$

where $b=L_{2} / L_{1}$. Figure 7 shows the data collapse obtained when the time scale of the system of size $L_{1}$ is rescaled by a factor $\left(L_{2} / L_{1}\right)^{z}$. The results obtained by rescaling lattices of sizes $81 / 27,243 / 81$, and $729 / 243$ are $z=2.76(2), 2.65(1)$, and 2.60(3), respectively. So we observed a systematic decrease in $z$ when the segmentation step of the fractal is increased, and consequently it is no longer valid to set a single value of the dynamic exponent for all segmentation steps. This observed behavior is similar to an observation reported previously [9] where the fixed point intersection of the Binder cumulant for different sizes was replaced by a se-

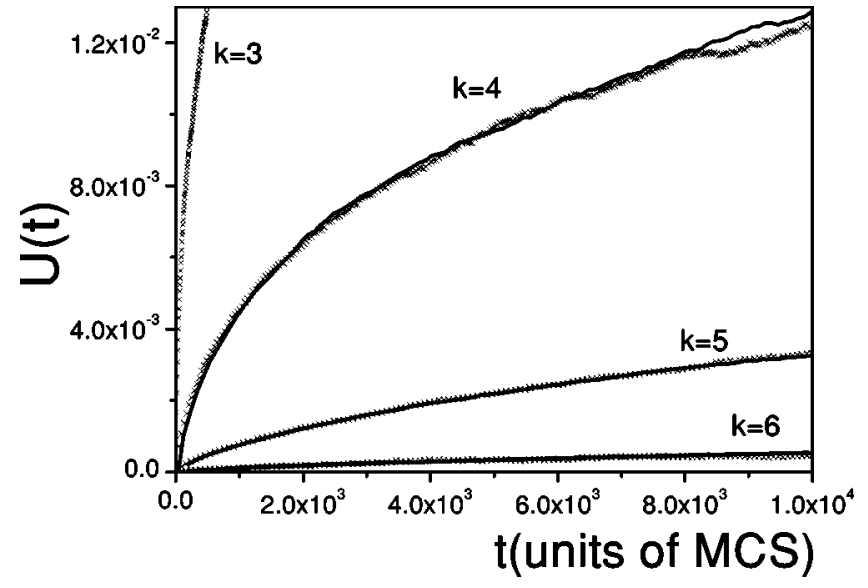

FIG. 8. Scaling plots of the second-order Binder cumulant obtained using data corresponding to adjacent pairs of segmentation steps $\left(k_{i}, k_{i+1}\right)$ with sizes $\left(L_{i}, L_{i+1}\right)$, respectively. The crosses (solid lines) correspond to systems of sizes $L_{i+1}\left(L_{i}\right)$. The time scale for the latter is rescaled by a factor $\left(L_{i+1} / L_{i}\right)^{z}$ in order to achieve superposition to the former; in each case the exponent $z$ is taken as a fitting parameter. Data obtained by averaging over 720, 17 000, 47000 , and 70000 different samples, for $k=65,4$, and 3, respectively.

quence of intersection points occurring at "effective" critical temperatures, while the actual critical temperature was defined as the limit for $k \rightarrow \infty$ (Fig. 8).

Summing up, the FSS method applied to the dynamic behavior of Binder cumulant only allows us to establish an upper bound to the dynamic exponent given by $z=2.60(3)$.

In order to obtain an independent estimation of the dynamic exponent $z$ that does not involve calculations with segmentation steps smaller than 6 we study the scaling behavior of the time correlation function:

$$
C(r, t)=\left[\left\langle\frac{1}{N} \sum_{i=1}^{N} s_{i}(t) s_{i+r}(t)\right\rangle\right]
$$

where $i+r$ indicates a site displaced by $r$ lattice spacings relative to site $i$. Our purpose is to study the onset of correlations between spins when an initially completely disordered system $(T=\infty)$ has been quenched to $T=T_{c}$. Conventional critical scaling implies the following scaling form for $C(r, t)$ :

$$
C(r, t)=r^{-(d-2+\eta)} f_{c}(r / \xi(t))
$$

Assuming that the hyperscaling relation given by $d_{\text {eff }}$ $=2 \beta / \nu+\gamma / \nu$ holds for this system, and using $\eta=2-\gamma / \nu$, we may replace $d-2+\eta$ in Eq. (11) by $2 \beta / \nu$. As $2 \beta / \nu z$ has already been obtained directly in the simulations from the decay of the magnetization from the ordered state [Eq. (6)], and $\xi(t)$ is expected to behave as $t^{1 / z}$, we may plot 


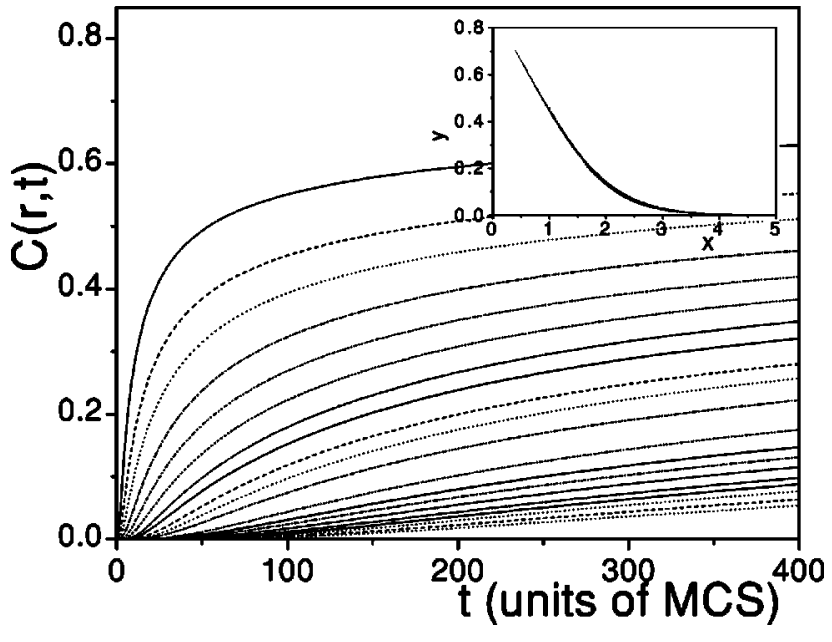

FIG. 9. Time correlation functions $C(r, t)$ for $r$ ranging from $r$ $=\sqrt{17}$ (upper curve) to $r=\sqrt{530}$ (lower curve) obtained for the $\mathrm{SC}(3,1)$ and $k=6$. The inset shows plots of the scaled correlation $y=r^{(2 \beta / v z) z} C(r, t)$ as a function of the scaled variable $x=r / t^{1 / z}$, for $z=2.55$ and $2 \beta / \nu z=0.0682$. We have taken the value of $2 \beta / \nu z$ from the decay of $M(t)$ obtained in this work and adjusted the value of $z$ that gives the best collapse of the curves. For $z=2.50$ and 2.60 the curves show noticeable deviations from the collapsed form (not shown here for the sake of space).

$$
r^{(2 \beta / v z) z} C(r, t) \text { vs } \frac{r}{t^{1 / z}},
$$

and look for the value of $z$ that make the curves to collapse. This procedure has been applied by Humayun and Bray [25] to obtain $z$ for the Ising model for $d=2$.

In Fig. 9 we show plots of $C(r, t)$ as a function of $t$ obtained for different values of $r$ ranging from 4 to 23. The inset shows the best collapse of the curves obtained for $z$ $=2.55$. This value was obtained by performing a fit of the scaled data to a four-parameter function given by

$$
f(x)=m x+b-m \pi^{-1 / 2} \int_{0}^{x} \int_{-\infty}^{(u-\alpha) / \sigma} e^{-v^{2}} d u d v,
$$

which we have empirically found to fit the data quiet well. We have also checked that other functional dependences for $f(x)$ that also fit the data only modify the value of $z$ by less than 0.01 . The obtained value for $k=6$, i.e., $z=2.55(1)$, is consistent with the trend observed from the FSS analysis of Binder's cumulant and sets our upper bound for the dynamic exponent.

The relationship $d_{\text {eff }} / z=0.693$ has been determined quite accurately by using three different kinds of measurements: the Binder cumulant, the slope of the second moment of the order parameter, and the autocorrelation function (see Table III). So, taking $z=2.55$ the effective dimension becomes $d_{\text {eff }} \simeq 1.77$, i.e., a figure that is noticeably smaller than the Haussdorf dimension $d_{H}=1.89$ of the $\mathrm{SC}(3,1)$. It should be noted that previous estimations using FSS studies gave values of $d_{\text {eff }}$ very close to $d_{H}$ (for a compilation of published results see Table I). However, Pruessner et al. [10] have estimated $d_{\text {eff }} \simeq 1.7$, also smaller than the Haussdorf dimension.
On the other hand, using the value $1 / \nu z=0.282$ determined from the slope of the logarithmic derivative of the order parameter [see Eq. (7) $]$ and $z=2.55$, our estimation for the lower bound of the correlation length exponent becomes $\nu \simeq 1.39$, which is significantly larger than the exact value of the Ising model given by $\nu=1$.

\section{DISCUSSION AND CONCLUSIONS}

It is shown that the short-time dynamics approach is a powerful method for the study of the critical behavior of the Ising model embedded in a fractal structure, where the translational symmetry is changed for the scale invariance. This method allows us to obtain a self-consistent determination of the critical temperature and the complete set of the critical exponents. This self-consistency is achieved by using three different initial conditions for the study of the dynamics.

The critical temperature determined in the present work for $k=6$ (an upper bound for this system) is in agreement with the value reported by Prussener et al. [10], which was obtained from equilibrium measurements. We note that these authors have used the slope method, a procedure that is free of the finite-size effects involved in FSS calculations. A critical discussion of the values reported by other authors employing different techniques is presented in the discussion of the results listed in Tables I and II.

The exponent $\theta$ of the initial increase in $M(t)$ determined for the segmentation step $k=6$ and extrapolated to the $m_{0}$ $\rightarrow 0$ limit is slightly smaller than the value corresponding to the two dimensional Ising model (see Table I). Our result is also in agreement with that reported by Zheng and Li [11], obtained by locating the spins at the vertices of the $\operatorname{SC}(3,1)$ and for the same segmentation step. In this way, these results suggest that the fractal structure does not significantly affect the exponent $\theta$.

The dynamic exponent $z$ has been obtained by means of two independent measurements. Binder's cumulant method allows us to determine the decreasing trend of $z$ when $k$ is increased, so we obtained an upper bound given by $z=2.60$. Further analysis of correlation functions allows us to improve the estimation of this upper bound, which is given by $z=2.55$.

Our value for the exponent $\beta=0.121$ of the order parameter for the segmentation step $k=6$ is also in agreement with the trend of the results reported by Pruessner et al. [10] for segmentation steps $k=4$ and 5 (see Table II), suggesting that our estimation can be taken as a lower bound. This value is only slightly smaller than the exact exponent corresponding to the 2D Ising magnet, namely, $\beta=0.125$. So, we should not disregard the possibility that for $k \rightarrow \infty$ the order parameter critical exponent may adopt the same value for both systems.

On the other hand, our estimations of the exponents $\gamma$ $=2.22$ of the susceptibility and $\nu=1.39$ of the correlation length, are significantly larger than those obtained for the 2D Ising system, namely, $\gamma=1.75$ and $\nu=1$, respectively. Observing the trend reported by Pruessner et al. [10] (see Table II) it is expected that our estimation for $\gamma$ could be taken as a lower bound. Also, $\nu$ may be taken as a lower bound because it is evaluated from the measurement of $\nu z$ that in- 
volves our estimation of an upper bound for $z$.

The relationship $d_{\text {eff }} / z=0.693$ has been determined quite accurately; then taking $z=2.55$ we conclude that $d_{\mathrm{eff}} \sim 1.77$ $<d_{H}$.

Finally we would like to remark that due to the huge statistic achieved in the evaluation of the dynamic properties, a soft oscillation around the power-law decay of the magnetization was observed at criticality (see Fig. 2). The same oscillation was also observed for the disordered initial state in the behavior of the autocorrelation (see Fig. 7). The oscillation can clearly be detected by subtracting the fitted power law from the actual data, as shown in Fig. 10. This oscillation is very nicely reproduced in both measurements (up to $t \simeq 2 \times 10^{3}$ in Fig. 10) and, to the best of our knowledge, this is the first evidence reported about this interesting behavior of the dynamic properties of the Ising model in a fractal substrate. We have clear signs that the observed oscillations are related to the topological properties of the fractal lattice. A more detailed investigation of these oscillations for the $\mathrm{SC}(3,1)$ and other fractals will be published elsewhere [26].

We would finally like to note the self-consistency of our results obtained by the application of the STD method to the study of the critical behavior of the Ising model on fractal structures. However, it should be recognized that there are still discrepancies in the values of the critical exponents when STD results are compared to those obtained using standard finite-size scaling of equilibrium data. For example, in contrast to our data, recent FSS results of Carmona et al. [7] and Monceau and Perreau [9] lead to an effective dimension almost equal to the Hausdorff one. So we conclude that the origin of the discrepancies may be related to the fact that the critical behavior of the Ising magnet on fractal substrates is very particular, since it is linked to the dependence of most

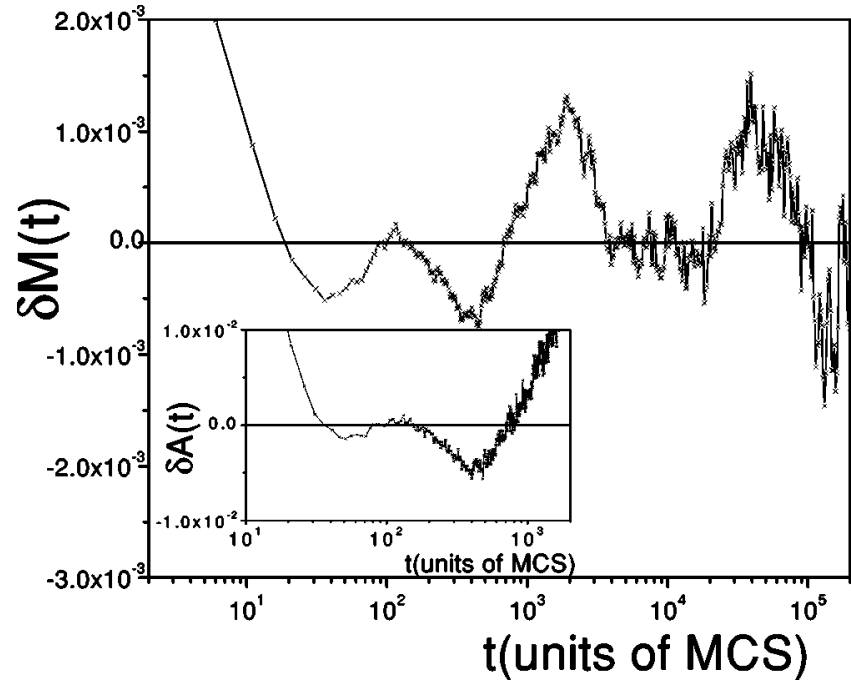

FIG. 10. Linear-log plots of the difference between the actual data corresponding to the decay of the order parameter obtained starting with $m_{0}=1$ and the best power-law fit of the curve versus time. The inset shows a similar plot but corresponding to the autocorrelation measured starting with $m_{0}=0$.

physical observables upon the number of iteration steps of the structure.

\section{ACKNOWLEDGMENTS}

This work was supported by CONICET, UNLP, ANPCyT, and Fundación Antorchas (ARGENTINA). The A. von Humboldt Foundation (Germany) is greatly acknowledged for the provision of valuable computer equipment. The authors thank Silvio Franz for fruitful discussions.
[1] Fractals and Disordered Sytems, edited by A. Bunde and S. Havlin (Springer-Verlag, Berlin, 1991).

[2] Fractals in Science, edited by A. Bunde and S. Havlin (Springer-Verlag, Berlin, 1995).

[3] Y. Gefen, B. B. Mendelbrot, and A. Aharony, Phys. Rev. Lett. 45, 855 (1980).

[4] Y. Gefen, A. Aharony, and B. B. Mendelbrot, J. Phys. A 16, 1267 (1983).

[5] Y. Gefen, A. Aharony, and B. B. Mendelbrot, J. Phys. A 17, 1277 (1984).

[6] B. Bonnier, Y. Leroyer, and C. Meyers, Phys. Rev. B 40, 8961 (1989).

[7] J. M. Carmona, U. M. B. Marconi, J. J. Ruis-Lorenzo, and A. Tarancón, Phys. Rev. B 58, 14387 (1998).

[8] P. Monceau, M. Perreau, and F. Hébert, Phys. Rev. B 58, 6386 (1998).

[9] P. Monceau and M. Perreau, Phys. Rev. B 63, 184420 (2001).

[10] G. Pruessner, D. Loison, and K. D. Schotte, Phys. Rev. B 64, 134414 (2001).

[11] G. P. Zheng and Mo Li, Phys. Rev. E 62, 6253 (2000).
[12] J. C. Le Guillou and J. Zinn Justin, J. Phys. (Paris) 48, 19 (1987).

[13] L. Hao and Z. R. Yang, J. Phys. A 19, 1627 (1987).

[14] P. Monceau and P. Y. Hsiao, Physica A 331, 1 (2004).

[15] P. Monceau and P. Y. Hsiao, Phys. Lett. A 300, 687 (2002).

[16] P. Hsiao and P. Monceau, Phys. Rev. B 67, 064411 (2003).

[17] H. K. Janssen, B. Schaub, and B. X. Schmittmann, Z. Phys. B: Condens. Matter 73, 539 (1989).

[18] B. Zheng, M. Schulz, and S. Trimper, Phys. Rev. Lett. 82, 1891 (1999).

[19] B. Zheng, Int. J. Mod. Phys. B 12, 1419 (1998).

[20] L. Schülke and B. Zheng, Phys. Lett. A 215, 81 (1996).

[21] I. Vattulainen, T. Ala-Nissila, and K. Kankaala, Phys. Rev. E 52, 3205 (1995).

[22] M. E. J. Newman and G. T. Barkema, Monte Carlo Methods in Statistical Physics (Clarendon Press, Oxford, 2001).

[23] P. Grassberger, Physica A 214, 547 (1995).

[24] B. Zheng, Physica A 283, 80 (2000).

[25] K. Humayun and A. J. Bray, J. Phys. A 24, 1915 (1991).

[26] M. A. Bab, G. Fabricius, and E. V. Albano (unpublished). 